\title{
Intelligent Control for Pneumatic Servo System
}

\author{
Jinhua LI, Yoshiki MIZUKAMI, Kanya TANAKA \\ Department of Electrical and Electronic Engineering \\ Faculty of Engineering \\ Yamaguchi University \\ 2-16-1 Tokiwadai, Ube, 755-8511, Japan \\ Tel: 0836-85-9430 \\ E-mail:ktanaka@eee.yamaguchi-u.ac.jp
}

\begin{abstract}
Internal model control (IMC) has a number of advantages for enhancing control performance. IMC can minimize disturbance greatly. IMC is attractive for industrial users because it has only one tuning parameter. In this paper, we propose an intelligent control method in which IMC control is combined with neural networks (NN). NN is used to get the suitable control parameter when the plant contains non-linear elements. We apply the proposed intelligent control method for a pneumatic servo system which usually contains non-linearity. The effectiveness of the proposed design method is confirmed by experiments using the existent pneumatic servo system.
\end{abstract}

\section{KEY WORDS}

Internal Model Control, Intelligent Control, Neural Networks, Pneumatic Servo System

\section{Internal Model Control}

A unifying review of the IMC-type schemes was first presented by Garcia and Morari (1982) [1]. IMC structure allows a direct controller design procedure where control quality and robustness can be influenced in a direct manner. IMC tuning rules have a number of advantages for improving controller performance. IMC minimize disturbance influence and use only one tuning parameter. The parameter relates directly to the closed-loop speed of response and to the robustness of the control loop; and the larger the tuning parameter, the greater the robustness.

Fig. 1 shows an IMC structure. Assume that the discrete-time model of the plant is $G\left(z^{-1}\right)$. Because IMC has to be stable and realizable, we introduce the following factorization

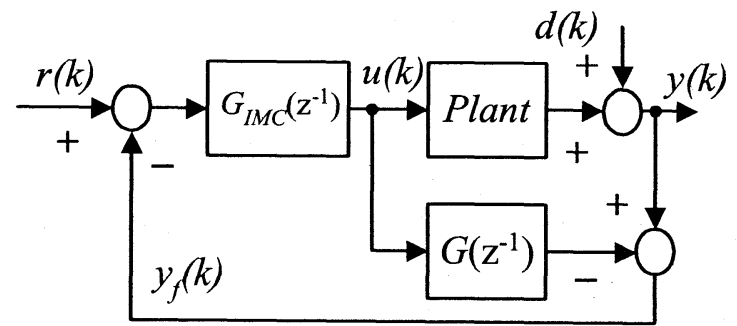

Fig. 1. An IMC structure. 


$$
G\left(z^{-1}\right)=P_{M}\left(z^{-1}\right): P_{A}\left(z^{-1}\right),
$$

where $P_{A}\left(z^{-1}\right)$ contains all the time delays and zeros outside the unit circle of the plant. If $\phi_{i}$ are the zeros outside the unit circle, then $P_{A}\left(z^{-1}\right)$ is given as

$$
P_{A}\left(z^{-1}\right)=z^{-N} \prod_{i=1}^{h} \frac{\left(1-\phi_{i}^{-1}\right)\left(1-\phi_{i} z^{-1}\right)}{\left(1-\phi_{i}^{-1} z^{-1}\right)\left(1-\phi_{i}\right)},
$$

where $N$ is the shift due to the time delay and $h$ is the number of zeros outside the unit circle. Note that $P_{A}(1)=1$.

Now the IMC controller $G_{I M C}\left(z^{-1}\right)$ is given by

$$
G_{I M C}\left(z^{-1}\right)=P_{M}^{-1}\left(z^{-1}\right) \cdot f\left(z^{-1}\right),
$$

where

$$
f\left(z^{-1}\right)=\frac{1-\alpha}{1-\alpha z^{-1}} .
$$

If $G\left(z^{-1}\right)$ has negative zeros inside the unit circle, the equivalent $G_{I M C}\left(z^{-1}\right)$ will have negative poles which result in ringing. The digital controller exhibits ringing in the controlled variable response. Use a pole at the origin for each zero of $G\left(z^{-1}\right)$ with negative real part, then the controller is now given as,

$$
G_{I M C}\left(z^{-1}\right)=G_{I M C 0}\left(z^{-1}\right) \frac{z^{-p}}{\prod_{i=1}^{p}\left(1-\phi_{i}\right)},
$$

where $G_{I M C 0}\left(z^{-1}\right)$ is the original controller used without the negative poles.

\section{IMC for Pneumatic Servo System}

We assume that the discrete-time plant model of the pneumatic servo system is as following [2]

$$
y(k)=\frac{z^{-2} \cdot B\left(z^{-1}\right)}{A\left(z^{-1}\right)} \cdot u(k),
$$

$$
\begin{aligned}
& A\left(z^{-1}\right)=1+a_{1} z^{-1}+a_{2} z^{-2}+a_{3} z^{-3}, \\
& B\left(z^{-1}\right)=b_{0}+b_{1} z^{-1}+b_{2} z^{-2} .
\end{aligned}
$$

Assume that the model have two zeros inside of the unit circle. One of them is positive, and another is negative. We assume that the zeros inside the unit circle are $Z_{1}$ and $Z_{2}, Z_{1}$ is positive, and $Z_{2}$ is negative. We know that $Z_{2}$ is the problematic zero. As explained in section 1,

$$
G_{I M C 0}\left(z^{-1}\right)=\frac{A\left(z^{-1}\right)}{b_{0} z^{-1}\left(1-Z_{1} z^{-1}\right)} \cdot \frac{1-\alpha}{1-\alpha z^{-1}} .
$$

Now, we can get the IMC controller from (5) as following

$$
G_{I M C}\left(z^{-1}\right)=G_{I M C 0}\left(z^{-1}\right) \cdot \frac{z^{-1}}{1-Z_{2}} .
$$

From Fig. 1, we have

$$
u(k)=G_{I M C}\left(z^{-1}\right) \cdot\left[r(k)-y_{f}(k)\right],
$$

where

$$
y_{f}(k)=y(k)-G\left(z^{-1}\right) \cdot u(k) .
$$

\section{NN based IMC}

In Fig. 1, we define

$$
e_{f}(k)=r(k)-y_{f}(k) \text {. }
$$

From (9) to (13), the discrete-time IMC can be written as following

$$
\begin{aligned}
u(k)= & \left(\alpha+Z_{1}\right) \cdot u(k-1)-\alpha \cdot Z_{1} \cdot u(k-2) \\
& +\frac{1-\alpha}{b_{0}\left(1-Z_{2}\right)}\left[e_{f}(k)+a_{1} \cdot e_{f}(k-1)\right. \\
& \left.+a_{2} \cdot e_{f}(k-2)+a_{3} \cdot e_{f}(k-3)\right] .
\end{aligned}
$$

If we define

$$
\begin{aligned}
E_{f}(k) & =e_{f}(k)+a_{1} \cdot e_{f}(k-1) \\
& +a_{2} \cdot e_{f}(k-2)+a_{3} \cdot e_{f}(k-3),
\end{aligned}
$$

then (14) can be written as

where

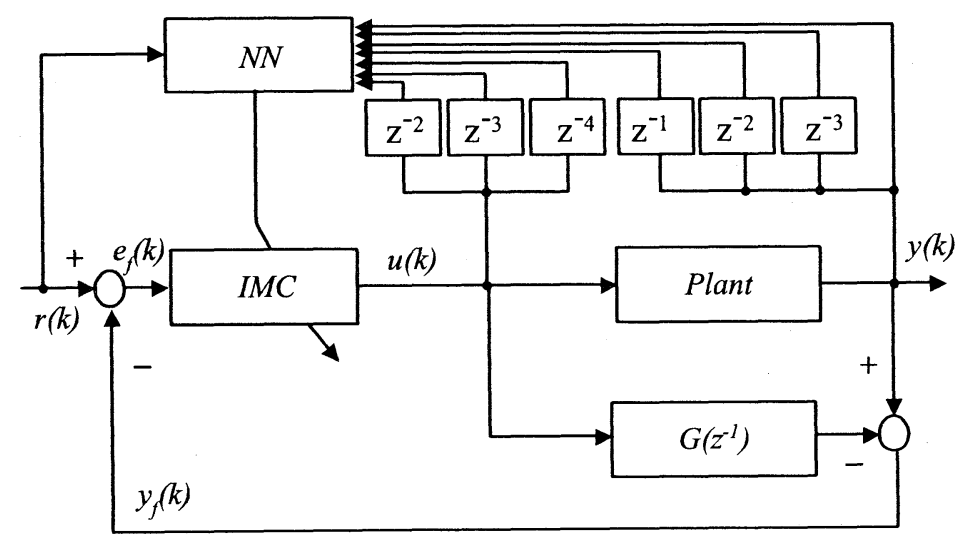

Fig. 2. Block diagram of NN based IMC. 


$$
\begin{aligned}
u(k)= & \left(\alpha+Z_{1}\right) \cdot u(k-1)-\alpha \cdot Z_{1} \cdot u(k-2) \\
& +\frac{1-\alpha}{b_{0}\left(1-Z_{2}\right)} E_{f}(k) .
\end{aligned}
$$

For more rapid closed-loop speed of response, we can decrease the " $\alpha$ ". For more robust response, we can increase the " $\alpha$ ". But, it is not so easy to select a proper " $\alpha$ " for a specific system, especially for a nonlinear system. So, we introduce NN to do it. The NN will find a proper " $\alpha$ " after learning. The applications of $\mathrm{NN}$ in IMC were introduced in [3] and [4]. In [3] and [4], the author trained $\mathrm{NN}$ to represent the plant response and the inverse of the plant. The scheme in this paper is different from ones in [3] and [4]. Fig. 2 is the block diagram of $\mathrm{NN}$ based IMC controller proposed in this paper. Fig. 3 is the architecture of NN. A 3-layer NN with a structure of $p-q-1$ is used. $p, q$ and 1 represent the number of nodes in each layer.

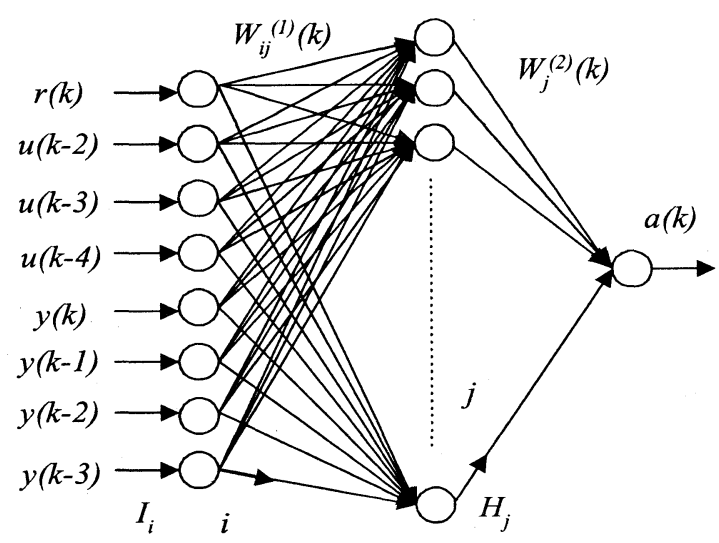

Fig. 3. Architecture of the NN.

The inputs of the input layer are: $I_{i}(k)=\{r(k)$, $u(k-2), u(k-3), u(k-4), y(k), y(k-1), y(k-2)$, $y(k-3)\}$. The output of the output layer is " $\alpha(k)$ ". $W_{i j}^{(1)}(k)$ are the weights from node $i$ of the input layer to node $j$ of the hidden layer. $W_{j}^{(2)}(k)$ are the weights from node $j$ of the hidden layer to the output " $\alpha(k)$ ". The output of the node $j$ of the hidden layer is

$$
H_{j}(k)=f_{s}\left(\sum_{i=1}^{p} W_{i j}^{(1)}(k) \cdot I_{i}(k)\right) \text {. }
$$

And the output of the output layer is

$$
\alpha(k)=f_{s}\left(\sum_{j=1}^{q} W_{j}^{(2)}(k) \cdot H_{j}(k)\right)
$$

where $f_{s}(\cdot)$ is the following sigmoid function

$$
f_{s}(x)=\frac{1}{1+e^{-x}} \text {. }
$$

The weights are updated to minimize the following object function

$$
J=0.5 \cdot[e(k)]^{2},
$$

where

$$
e(k)=r(k)-y(k) \text {. }
$$

The weights $W_{j}^{(2)}(k)$ are updated as following

$$
W_{j}^{(2)}(k+1)=W_{j}^{(2)}(k)+\Delta W_{j}^{(2)}(k),
$$

where

$$
\begin{aligned}
\Delta W_{j}^{(2)}(k) & =-\eta \frac{\partial J}{\partial W_{j}^{(2)}(k-2)} \\
& =-\eta \frac{\partial J}{\partial y(k)} \cdot \frac{\partial y(k)}{\partial u(k-2)} \cdot \frac{\partial u(k-2)}{\partial a(k-2)} \cdot \frac{\partial a(k-2)}{\partial W_{j}^{(2)}(k-2)}
\end{aligned}
$$

The weights $W_{i j}^{(1)}(k)$ are also updated as

$$
W_{i j}^{(1)}(k+1)=W_{i j}^{(1)}(k)+\Delta W_{i j}^{(1)}(k),
$$

where

$$
\begin{aligned}
\Delta W_{i j}^{(1)}(k) & =-\eta \frac{\partial J}{\partial W_{i j}^{(1)}(k-2)} \\
& =-\eta \frac{\partial J}{\partial y(k)} \cdot \frac{\partial y(k)}{\partial u(k-2)} \cdot \frac{\partial u(k-2)}{\partial a(k-2)} \cdot \frac{\partial a(k-2)}{\partial W_{i j}^{(1)}(k-2)}
\end{aligned}
$$

From (6), (16) and (20), we have

$$
\begin{aligned}
& \frac{\partial y(k)}{\partial u(k-2)}=b_{0} \\
& \frac{\partial u(k-2)}{\partial \alpha(k-2)}=u(k-3)-Z_{1} u(k-4)-\frac{E_{f}(k-2)}{b_{0} \cdot\left(1-Z_{2}\right)},
\end{aligned}
$$

$$
\frac{\partial J}{\partial y(k)}=-[r(k)-y(k)] .
$$

Furthermore from (18) and (19), we have

$$
\frac{\partial a(k-2)}{\partial W_{j}^{(2)}(k-2)}=a(k-2)\{1-a(k-2)\} \cdot H_{j}(k-2) \text {. }
$$

Define

$$
\begin{aligned}
& \delta^{(2)}=b_{0} \cdot e(k) \cdot\left[u(k-3)-Z_{1} \cdot u(k-4)\right. \\
& \left.-\frac{E_{f}(k-2)}{b_{0} \cdot\left(1-Z_{2}\right)}\right] \cdot \alpha(k-2) \cdot[1-\alpha(k-2)],
\end{aligned}
$$

then (23) can be represented as

$$
\Delta W_{j}^{(2)}(k)=\eta \delta^{(2)} H_{j}(k-2) .
$$

Now, we use the same method to get $\Delta W_{i j}^{(1)}(k)$.

$$
\begin{aligned}
& \Delta W_{i j}^{(1)}(k)=\eta \delta^{(1)} I_{i}(k-2), \\
& \delta^{(1)}=\delta^{(2)} W_{j}^{(2)}(k-2) H_{j}(k-2)\left[1-H_{j}(k-2)\right] .
\end{aligned}
$$




\section{Experimental results}

We know the time delay of the system is about $40 \mathrm{~ms}$ from step response, so we choose the sampling time as $40 \mathrm{~ms}$. We adopt the constant trace algorithm as the identification method. The detail of the identification algorithm was introduced in the paper by one of the authors [2]. From identification results, we have the parameters in (7) and (8) as follows

$$
\begin{array}{ll}
a_{1}=-1.3, & a_{2}=-0.13, \quad a_{3}=0.44, \\
b_{0}=3.1, \quad b_{1}=-2.3, \quad b_{2}=-0.46 .
\end{array}
$$

We also find the zeros are $\mathrm{Z1}=0.906$ and $\mathrm{Z2}=-0.164$.

A rectangular wave is used as an object input. The amplitude of the rectangular wave is from $25[\mathrm{~mm}]$ to $-25[\mathrm{~mm}]$. The period of the rectangular wave is 40 seconds.

It is difficult that the plant output follows the rectangular wave during the period just after the sign of the object input is changed. The error of this period is no meaning for the learning of NN. So we stop the learning of $\mathrm{NN}$ during this period. We restart the leaning after this period. We design the special period as 200 sampling times.

Because of the non-symmetry of the response, it is difficult to get good learning results by using only one $\mathrm{NN}$ in both sides of the rectangular wave. So we use two $\mathrm{NN}$. One is for positive side and another is for negative side. From the experiment, we find that the suitable control parameter $\alpha(k)$ in positive side is 0.7137 , and in negative side is 0.4978 . But because of the parameter variation of the controlled plant, the IMC parameter will be different slightly in every time. And this is just the advantage of the proposed method. It means that the control parameter is not fixed, the proper control parameter will be found by $\mathrm{NN}$ after on-line learning.

From the figure of response (Fig. 4), we can find that the using of $\mathrm{NN}$ based IMC can give desired servo tracking. $\mathrm{NN}$ can find suitable control parameter after on-line learning. And the response shows no overshoot. From the histogram of the positioning error (Fig. 5 and Fig. 6), we can find, NN can reduce the positioning error. The steady-state positioning error is less than $\pm 0.07 \mathrm{~mm}$.

\section{Conclusions}

A novel NN based IMC control scheme for the pneumatic servo system is proposed. IMC tuning method uses only one parameter. The parameter relates directly to the closed-loop speed of response and to the robustness of the control loop. NN has the ability of on-line learning. $\mathrm{NN}$ is used to get the suitable control parameter of IMC after on-line learning. This makes the system have robustness and adaptive capability. The effectiveness of the proposed design method is confirmed by experiments using the existent pneumatic servo system.

\section{References}

1. Garcla,C. E., and M. Morari, "A Unifying Review and Some New Results”, Ind. Eng. Chen. Proc. Des. Dev., 1982, 21, (p308-p323)

2. Kanya Tanaka, "Improved Design Scheme of MRAC for Pneumatic Servo System with Additive External Forces", Transactions of the Japan Society of Mechanical Engineers (C), Vol. 63, (613), 1997,9, (p3110-p3116)

3. Hunt K., Sbarbaro D., "Neural networks for nonlinear internal model control", IEE Proc.-D, Vol. 138(5), 1991, 99, (p431-p438)

4. Isabelle Rivals, etc., "Internal Model Control Using Neural Networks", Proceeding of the IEEE Int. Sym. on Ind. Elec., June, 1996, (p17-p20)

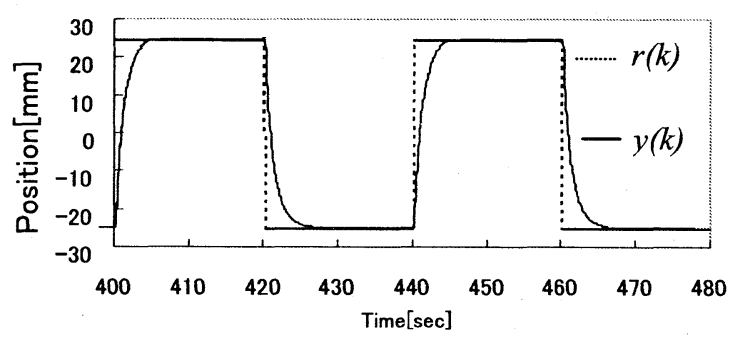

Fig. 4. Responses of NN based IMC.

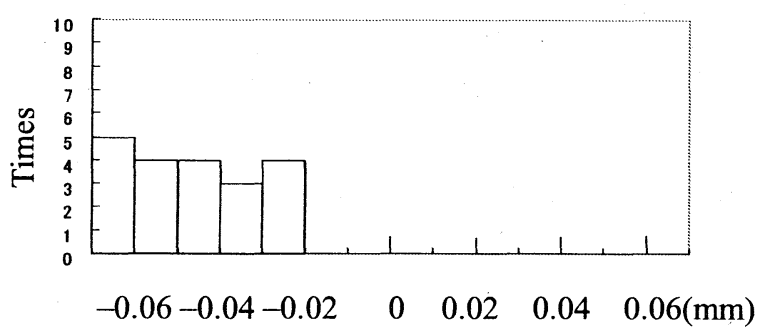

Fig. 5. Positioning error of positive side $(\alpha=0.7137)$

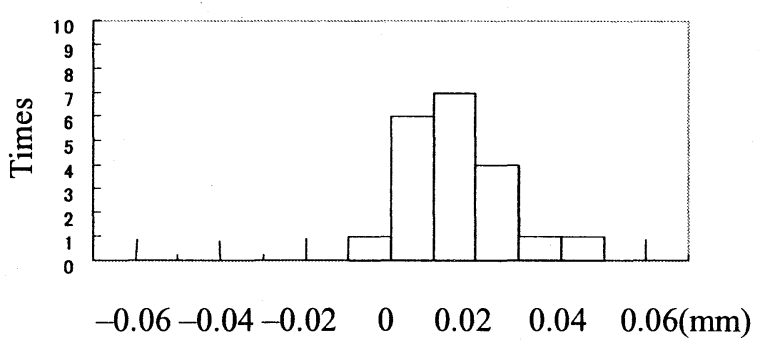

Fig. 6. Positioning error of negative side ( $\alpha=0.4978$ ) 